\title{
Zur Frage der kongenitalen Anlage bei pluriglandulärer Insuffizienz.
}

\author{
Von \\ Prof. Hans Curschmann (Rostock).
}

(Aus der Med. Univ.-Poliklinik Rostock.)

(Eingegangen am 28. Juni 1920.)

$\mathrm{Krabbe}^{1}$ ) berichtet im 55 . Band dieser Zeitschrift über 2 Fälle von früherworbener oder kongenitaler pluriglandulärer Insuffizienz, bemerkt einleitend, daß die meisten bisher veröffentlichten Fälle bei Erwachsenen oder größeren Kindern nach Infektion oder Intoxikationen (also exogenen Anlässen) entstanden seien, und schreibt dann, es sei ihm nicht gelungen, abgesehen von Kretinismus, in der Literatur Fälle zu finden, die kongenital oder in früher Kindheit entstandene pluriglanduläre Insuffizienz darstellten.

Es scheint also dem dänischen Autor entgangen zu sein, daß in der einschlägigen deutschen Literatur für die meisten Formen dieses vielgestaltigen Krankheitsbildes eine kongenitale Anlage, nicht nur im anatomisch-histologischen Sinne, sondern auch in klinisch nachweisbarer Form angenommen wird. J. Ba uer ${ }^{2}$ ) betont, daß eine besondere konstitutionelle Beschaffenheit des Organismus für das Zustandekommen der chronischen Blutdrüsenerkrankungen erforderlich sei. Wiesel und Goldstein haben diese endogene Grundlage für die pluriglanduläre Insuffizienz in einer generellen oder solitären Hypoplasie und Bindegewebsdiathese der endokrinen Drüsen angenommen. $\mathrm{Ob}$ diese Annahme zutrifft, oder, ob andersartige somatische oder funktionelle Anomalien einzelner Drüsen oder des ganzen Systems (mit Vorherrschen einer oder einiger Drüsen im Bilde der Dyshormonie) die kongenitale Grundlage für die pluriglanduläre Insuffizienz bilden, möchte ich mit Bauer unentschieden lassen. Jedenfalls muß Krabbe gegenüber betont werden, daß kongenitale Hypo- und Dysplasien einzelner endokriner Drüsen im Krankheitsbild der später an multipler Blutdrüsensklerose erkrankenden Individuen recht häufig sind, wenn auch Fälle von so hochgradiger, von Geburt an vielfältiger endokriner

1) Diese Zeitschr. 55, 254 f. 1920.

2) Die konstitutionelle Disposition zu inneren Krankheiten. Berlin 1917. 
Drüseninsuffizienz, wie die Fälle von Krabbe, tatsächlich zu den Seltenheiten gehören. UUbrigens hatten bereits die Schöpfer des Begriffes der pluriglandulären Insuffizienz, Cla ude und Gougerot, eine ab origine minderwertige Anlage des Blutdrüsensystems angenommen (zit. nach J. Bauer). Auch der Beziehungen, die Wiesel ${ }^{1}$ ) zwischen dem Status thymo-lymphaticus und dem Infantilismus, der Asthenie und der pluriglandulären Insuffizienz konstatierte, sei hier gedacht.

Goldstein ${ }^{2}$ ) hat Fälle von familiärem Eunuchoidismus und Adipositas beschrieben, bei denen die Annahme der Hypoplasie mehrerer Blutdrüsen gerechtfertigt schien. Es gilt das zweifellos nicht für alle, aber doch für nicht wenige Formen des angeborenen Hypogenitalismus, der überhaupt als häufigstes, nicht selten monolokuläres Zeichen einer endokrinen Hypoplasie bei den mannigfachsten Krankheitsbildern, auch solchen, die nicht zur typischen pluriglandulären Insuffizienz im engeren Sinne gehören, ihr aber artverwandt sind, beobachtet wird.

Daß auch vorübergehende endokrine Störungen bei solchen Individuen, zumal, wenn sie sich noch in Entwicklung befinden, auftreten können, wie Tetanie und Myxödem, hat $\mathrm{Falta}^{3}$ ) hervorgehoben. Meine Beobachtungen bestätigen das besonders bezüglich der Tetanie.

Auch ich ${ }^{4}$ ) habe an drei mitgeteilten Fällen die angeborene Blutdrüsenschwäche hervorgehoben: Fall I, der Beziehungen zur Degeneratio genito-sclerodermatica v. Noordens hatte (mit Xanthosis), war von Geburt an schwächlich, war stets hypomenorrhoisch und frigide, Fall II, mit familiärem Ikterus einhergehend, hatte ein Geburtsgewicht von $4^{1} / 2$ Pfund, behielt infantile Stimme, hatte keinen Bartwuchs, machte mit 12 Jahren - ganz im Sinne der obigen Faltaschen Ausführungen einen flüchtigen Diabetes durch; Fall III, ein Fall mit Hypothyreose, Späteunuchoidismus mit Addisonsyndrom, ganz dem Typus von Cla ude und Gougerot entsprechend, war von Kind an ebenfalls nicht nur sehr schwächlich, sondern auch mit körperlichen und seelischen Stigmen des Feminismus behaftet. Es ist sehr bemerkenswert daß im Fall I und III, in dem später grob organische Atrophie der Keimdrüsen auftrat, bereits seit der Pubertät eine funktionelle Minderwertigkeit in Gestalt sexueller Frigidität bestanden hatte, die geradezu als Vorbote der später an denselben Organen lokalisierten anatomischen Degeneration auftrat.

1) Handbuch der Neurologie von Lewandows ky. Bd. 4. 1918.

2) Münch. med. Wochenschr. 1912, Nr. 47 und Arch. f. Psych. u. Nervenheilk. 53. 1914.

3) Lewandows kys Handbuch der Neurologie 4.

4) Zeitschr. f. klin. Med, 87. H. 1 u. 2. 
Eine angeborene hypogenitale Anlage mit einer ebenfalls angeborenen Parotishyperplasie finden wir als Vorboten der späteren pluriglandulären Insuffizienz ebenfalls bei dem folgenden Fall:

O. J., 47 jähr. Klempner. Familienanamnese o. B. Wurde angeblich, bis auf eine seit Geburt bestehende doppelseitige Parotisschwellung, normal geboren, hat sich körperlich und geistig leidlich gut entwickelt, hatte aber von Anfang an auffallend dünnen Haarwuchs und desgleichen Augenbrauen. Der Stimmwechsel trat zur üblichen Zeit nicht ein und ist überhaupt ausgeblieben. Die Libido sexualis blieb bis zum 26. Jahr völlig aus und war seitdem auch sehr gering. Nennenswerter Bartwuchs ist nie aufgetreten. Mit 36 Jahren Heirat, Coitus sehr selten; 1 gesundes Kind. Vor 6-7 Jahren Sehstörungen. 1915 Kataraktoperation, seit 1917 eigentümliche sklerosierende Keratitis des anderen Auges. Seitdem allgemeiner Rückgang der Kräfte, Schwellung, später Hartwerden der Füße und Unterschenkel.

Befund: Magerer, elender Mann, allgemeine Dürftigkeit der Muskulatur, Haut des Gesichts fahl, zum Teil atrophisch, nicht hart, desgleichen am Körper keine sklerodermische Haut; die Haut des unteren Drittels der Unterschenkel und der Füße glatt, außerordentlich hart, von der Unterlage nicht abhebbar, teils rötlich-livide, teils bräunlich pigmentiert.

Kopfhaar sehr dünn, stränig, fahl, zum Teil ergraut; Kinn- und Backenbart fehlt; einige wenige, kurze, weiße Schnurrbarthaare. Stamm- und Achselhaare fehlen. Stimme hoch, knabenhaft. Kehlkopf auffallend klein. Von der Schilddrüse nur ein kleiner Teil des mittleren Lappens, ziemlich derb, tastbar. Ohrläppchen angewachsen, mangelhafte Ausbildung der Helixfurchen.

Hirn- und Gesichtsschädel asymmetrisch, sonst o. B. Sella turcica im Röntgenbild o. B. Beiderseits starke (angeborene) Verdickung der Parotis; Tränendrüsen o. B. Sonst keine Drüsenschwellungen.

Rechts Iridektomie, links eigenartige, sklerosierende Keratitis.

Lungen o. B. Herz normal begrenzt, reine Töne. Puls etwa rigide. Blutdruck gesteigert, $170: 80 \mathrm{~mm} \mathrm{Hg}$.

Bauchorgane o. B.

Während die Extremitäten und der übrige Stamm sehr fettarm sind, sind Bauch und Schamhügel relativ fettreich; letzterer ist feminin geformt, das Scrotum sehr kurz bei relativer Breite, Penis sehr klein, linker Hoden klein, hart, rechter normal, beide auffallend hochstehend. Crines pubis sehr spärlich, feminin begrenzt.

Blut: $65 \%$ Hämoglobin, 4050000 Erythrocyten, 6100 Leukocyten, Färbeindex 0,9. Polynucleäre Leukocyten 62, Lymphocyten $34 \%$, eosinophile Leukocyten $2 \%$, UUbergangszahl $2 \%$; mikroskopisch Erythrocyten und Leukocyten o. B. Blutplättchen o. B.

Psyche: o. B., normal intelligent; keine affektive Störungen. Keine motorischen und sensiblen Störungen. Hirnnerven o. B. Alle Sehnenreflexe lebhaft; Fehlen aller Hautreflexe; kein Babinski. Chvostek und Trousseau fehlen.

Galvanische Prüfung des N. facialis, R. mentalis: KSZ. 2,6, ASZ. 4,0, AÖZ. 6,0 M.-A., des N. ulnaris: KSZ. 2,1, ASZ. 3,8, AÖZ. 5,0 M.-A.

Erbsches Phänomen also negativ.

Keine aktiven, mechanischen oder elektrisch-myotonischen Symptome, keine Zeichen von Myasthenie.

Von pluriglandulär bedingten Störungen sind also vorhanden: funktioneller und somatischer Hypogenitalismus, Fehlen der seḳundären Geschlechtsmerkmale, insbesondere Vox puerilis bei hypoplastischem 
Kehlkopf; Hypoplasie der Schilddrüse; symmetrische Sklerodermie beider Unterschenkel und Füße und eine Keratitis, die vielleicht dem sklerodermischen Prozeß verwandt ist; symmetrische Hyperplasie der Parotis; Katarakt.

Da eine deutliche Progredienz der meisten Symptome, insbesondere der allgemeinen Schwäche, besteht, ist an einer echten pluriglandulären Insuffizienz nicht zu zweifeln.

Wichtig ist, wie bemerkt, daß der Hypogenitalismus und die Parotishyperplasie kongenital waren, bzw. in frühester Kindheit bemerkt werden. Sie waren eben das Substrat der kongenitalen, hypoplastischen Anlage der Blutdrüsen, auf die sich dann nach langer postnataler Latenz (aus irgendwelchen exogenen Anlässen vielleicht) die fortschreitende, multiple Blutdrüsensklerose (vgl. Schilddrüse und Hoden!) aufbaute. Sehr bemerkenswert ist, da $\beta$ auch in diesem Falle die hypogenitale Anlage das Bild der angeborenen, lange latenten Hypoplasie beherrschte. Auch J. Ba uer, Goldstein u. a. heben die Bedeutung des Eunuchoidismus in diesem Sinne hervor. Es dürfte sich empfehlen, das Schicksal jugendlicher Eunuchoider auf etwaige spätere progressive pluriglanduläre Insuffizienz zu verfolgen. Ich glaube, daß dies die Zahl dieser Fälle sehr steigern würde.

Die angeborene Parotishyperplasie reiht sich, wie schon bemerkt, den übrigen Symptomen der pluriglandulären Insuffizienz ein. Die Beziehungen der Speicheldrüse zur Keimdrüse (insbesondere den Hoden) sind ja bekannt. Die Parotisvergrößerung bei Fettsucht zählt J. Ba uer zu den endokrinen Störungen und auch L. Moh $\mathrm{r}^{1}$ ) hebt die Zusammenhänge zwischen Parotis und Blutdrüsensystem hervor. Sehr bemerkenswert ist in diesem Sinne ein Fall J. Bauers mit Hodentumor, Parotishyperplasie und Struma, desgl. die von ihm zitierten Beobachtungen über familiäre und konstitutionelle Speicheldrüsenschwellung, die zum Teil als physiologische Variation gedeutet wurde. Jedenfalls muß man sagen, daß der Parotis künftig im Bilde der pluriglandulären Insuffizienz größere Aufmerksamkeit geschenkt werden muß als bisher, und gleichfalls den mit ihr ja auch im Mikuliczsehen Syndrom vereinigten Tränendrüsen. L. Mohr hatte übrigens bereits vor Jahren auf das Vorkommen dieses bisher als aleukämische Lymphadenose gedeuteten Syndroms im Rahmen innersekretorischer Symptombilder aufmerksam gemacht.

Die präsenile Katarakt ist ebenfalls ein bemerkenswertes Symptom endokriner Natur. Für die hypoparathyreoide Tetanie war die Bedeutung des Stars längst bekannt. Die wichtige Feststellung von A. Peters und seinen Schülern, daß viele Individuen mit Alterstar latente Tetaniesymptome aufweisen, bestätigte und verbreiterte den

1) Verhandl, d. Kongr. f. inn. Med. 1914, S. 476. 
Zusammenhang von Katarakt und Nebenschilddrüseninsuffizienz. Einen besonders bemerkenswerten Fall dieser Art aus der Petersschen Klinik sah ich neulich : einen älteren Mann mit doppelseitigem Altersstar, alternierenden Anfällen von Tetanie der Hände und „Bronchotetanie“", d. i. Anfällen von typischem, eosinophilem Asthma, nach dem ebenso, wie nach den Carpopedalkrämpfen, die Phänome von Chvostek und Erb exazerbierten; auch dieser Mann hatte doppelseitige Sklerodermie der Füße und Unterschenkel, die photographisch derjenigen des obigen Falles glich. Sonst bestanden keine Blutdrüsensymptome.

Star und Chvosteksches Phänomen sind nun recht häufig auch bei einer anderen Krankheit, deren pluriglandulärer Ursprung immer wahrscheinlicher wird ${ }^{1}$ ), bei der Dystrophia myotonjca ${ }^{2}$ ). Und auch bei ihr finden wir das oben so oft erwähnte Moment der kongenitalen sexualen Hypoplasie in Gestalt von Hodenhypoplasie, geschlechtlichem Infantilismus und - viel seltener - der Verminderung oder dem Fehlen der sekundären Geschlechtsmerkmale. Auch bei der dystrophischen Myotonie ist also die genitale Hypoplasie das angeborene Symptom der Blutdrüsenschwäche, die sich dann später auf Grund einer fixen familiären Krankheitsanlage zur weiteren, symptomatisch scharf umrissenen pluriglandulären Insuffizienz, d. h. der dystrophischen Myotonie ,auswächst".

Etwas ganz Analoges beobachtete ich auch bei der Myasthenia pseudoparalytica. Nachdem bereits von anderen Autoren angeborene Mißbildungen (z. B. Syndaktylie, Anomalien des Zentralkanals u. a.) beschrieben worden waren, konnte $\mathrm{ich}^{3}$ ) über zwei Frauen mit kongenitaler hochgradiger Hypoplasie der Genitalien und Daueramenorrhöe berichten, deren eine einen ausgesprochenen männlichen Gesamthabitus zeigte. Auch bei diesen Myastheniefällen war die sexuale Hypoplasie das angeborene Moment, zu dem sich nach einer postnatalen Latenz von fast drei Jahrzehnten die wirkliche Erkrankung, die fortschreitende Myasthenie, gesellte. Das ist im Rahmen dieser Mitteilung darum bemerkenswert, weil man bereits seit langem auch für die Myasthenie eine endokrine Genese vermutete. Ich er-

1) Tetanie und Star. Bonn 1898 und Lubarsch - Ostertag, Ergebn. 1914.

2) Hauptmann und neuerdings Nickau schreiben, daB ich auf Grund meiner negativen Untersuchungen betreffs Vagotonie und Sympathicotonie bei dystrophischer Myotonie auf die endokrinen Drüsen keinen Wert legte bzw. ihre genetische Wichtigkeit negiere. Das habe ich natürlich nicht getan, sondern sogar geschrieben: „Es ist dies negative Resultat natürlich keineswegs entscheidend für die Ausschließung einer innersekretorischen Störung bei der Dystrophia myotonica. Die anatomische Forschung wird... hier das letzte Wort zu sprechen haben" (Dtsch. Zeitschr. f. Nervenheilk. 53).

3) Dtsch. Arch. f. klin. Med. 85. 1906 u. Zeitschr. f. d. ges. Neur. u. Psych. 7, H. 3. 
innere an die zuerst von Lundborg ${ }^{1}$ ) und Chvostek jun. ${ }^{2}$ ) aufgestellte Theorie von einer chronischen Hyperfunktion der Nebenschilddrüsen, die ich bereits früher als sehr unwahrscheinlich bezeichnet habe. Neuere Beobachtungen bestätigen mir das. $\operatorname{Ich}^{3}$ ) fand bei zwei jungen Mädchen mit schwerer Myasthenie Chvosteksches Phänomen stark positiv, in einem der Fälle sogar mit galvanischer Übererregbarkeit (Erb), also Symptome, die unbedingt eher für eine Minderfunktion der Epithelkörperchèn sprechen, als für das Gegenteil. Ich erinnere auch daran, daß Tobias tetanieähnliche Anfälle bei Myasthenie beobachtet hat. Außer diesen endokrinen Störungen fand ich in beiden Fällen Strumen und Hyperplasie der Gl. thymi; beides, besonders das letztere, bekanntlich recht häufige Befunde bei dieser Erkrankung, so häufig, daß man auch an eine speziell thymogene Entstehung der Myasthenie gedacht hat. Endlich bedarf der Erwähnung, daß man auch ausgesprochenen M. Basedow bei Myasthenie beobachtet haben will. Einige Autoren haben auf Grund von klinischen Beobachtungen auch an die Hypophyse und die Nebennieren gedacht. Kurz es ist kein Zweifel, daß pluriglanduläre Störungen bei der Myasthenie eine Rolle spielen. Aus diesem Grunde ist im Hinblick auf die Krabbesche Arbeit die Tatsache, daß auch bei ihr angeborene Hypoplasien wichtiger endokriner Drüsen vorkommen, von großem Interesse, ebenso, wie der Umstand, daß sich bei ihr in zahlreichen Fällen eine Thymushyperplasie, die wir ja auch zu den streng konstitutionellen Stigmen zählen müssen, findet.

Diese wichtige Rolle pluriglandulärer Störungen bei der dystrophischen Myotonie und der Myasthenie erweckt heute darum erhöhtes Interesse, weil wir neuerdings durch die.Untersuchungen von $\operatorname{Frank}^{4}$ ), Harry Schäffer $r^{4}$ u. a. genauere Vorstellungen über das Wesen und die Form der sympathischen und parasympathischen Innervation des Muskelsystems, die ja, wie jede Funktion des autonomen Systems, unter dem bestimmenden Einfluß der endokrinen Drüsen stehen muß, gewonnen haben. Frank hat gezeigt, da $B$ in jedem quergestreiften Muskel gleichsam ein glatter, wahrscheinlich das Sarkoplasma, verborgen ist, das nicht vom motorischen Nervensystem, sondern vom autonomen innerviert wird. Den normalen Muskeltonus und gewisse krankhafte Steigerungen (Tetanus, Paralysis agitans) faßt Frank nicht als Funktion des Fibrillenapparates, sondern des autonom innervierten Sarkoplasmas auf. Schäffer wies dann nach, daß die sog. Tiegelsche Contractur des Muskels durch parasympathisch erregende

1) Dtsch. Zeitschr. f. Nervenheilk. 27, H. 3 u. 4.

2) Wiener klin. Wochenschr. 1908.

3) Diese Zeitschr. 50, $131 \mathrm{f}$.

4) Verhandl. d. Kongr. f. inn. Med. Dresden 1920. 
Mittel gefördert, durch sympathisch reizende und parasympathisch lähmende dagegen gehemmt wird.

Es liegt nun sehr nahe, die muskulären Erscheinungen der dystr. Myotonie und der Myasthenie, die ja bekanntlich durch Störungen im Bereich des zentralen und peripheren motorischen Neurons nicht erklärt werden können, auf die Funktionsveränderung der autonomen Innervation im obigen Sinne zu beziehen. Es sei daran erinnert, daß H. Päßler ${ }^{1}$ ) bereits vor Jahren auf Grund eines Vergleichs der experimentell von Joteyko gefundenen Sarkoplasmareaktion mit den galvanischen Muskelreaktionen seiner Fälle von dystr. Myot. zur Annahme kam, daß eine gesteigerte Erregbarkeit des Sarkoplas mas das Wesen der myotonischen Störung ausmachen solle. Der nervösen Beeinflussung dieser Sarkoplasmafunktionsänderung stand Päßler unter Ablehnung meiner ${ }^{2}$ ) Befürwortung einer zentral-nervösen Ätiologie - allerdings skeptisch gegenüber. Heute können wir die Päßlersche Theorie der Sarkoplasmaerregbarkeitssteigerung als fester fundiert ansehen, nachdem wir einerseits dessen autonome Innervation kennengelernt und andererseits die beträchtliche Rolle der das sympathische und parasympathische System regulierenden endokrinen Drüsen für die myot. Dystr. erkannt haben.

Für das - ja ebenfalls endokrin beeinflußte oder verursachte Bild der Myasthenie, die ja bezüglich des Mechanismus der Bewegungsstörung in vieler Beziehung der Myotonie direkt entgegengesetzte Züge zeigt, besteht deshalb wohl die Möglichkeit einer der Myotonie entgegengesetzten Funktionsstörung der autonomen Innervatoren des quergestreiften Muskels bzw. des Sarkoplasmas.

Weiter sei in diesem Zusammenhang noch des Hypoth yreoid is $\mathrm{m} u \mathrm{~s}$ gedacht, der in Gestalt des angeborenen Kretinismus allein von $\mathrm{Krab}$ be als kongenitale Form einer innersekretorischen Störung genannt wurde. Auch diese Mitteilung bedarf der Erweiterung. Einerseits gibt es Fälle von angeborener Thyreohypoplasie ohne Struma und allgemeine kretinoide Degeneration, darunter auch Fälle, die gleichzeitig mit Tetanie und Epilepsie verlaufen können, also den Symptomen der Epithelkörperhypoplasie. Andererseits gibt es Individuen mit eunuchoidem Habitus - aber ohne manifesten Hypothyreoidismus -, die erst im Erwachsenenalter auf exogene Reize hin (z. B. Unterernährung) an typischem Myxödem erkranken. Bei weiblichen $\mathrm{Pa}$ tienten sah ich dem letzteren dauernde Oligo- oder Amenorrhöe und Sterilität vorangehen. Auch beim Myxödem des Rückbildungsalters weisen bisweilen während des ganzen vorangegangenen Lebens mannigfache vasomotorische und sekretorische Störungen darauf hin, daß eine

1) Dtsch. Arch. f. klin. Med. 27.

2) Dtsch. Zeitschr. f. Nervenheilk. 45, 199. 
Zur Frage der kongenitalen Anlage bei pluriglandulärer Insuffizienz. 271

angeborene, konstitutionelle Blutdrüsenschwäche dem späteren Myxödem den Boden bereitet hat. Also muß auch beim Hypothyreoidismus des Erwachsenenalters mit einer angeborenen Unterentwicklung oder Unterfunktion endokriner Drüsen gerechnet werden. Eine ausführliche Darstellung des Verhältnisses der konstitutionellen Faktoren zum Hypothyreoidismus werde ich demnächst bringen und verweise auf $\operatorname{sie}^{1}$ ).

Zum Schluß erinnere ich daran, daß angeborene endokrine Störungen auch bei Rachitis, insbesondere der R. tarda, und Osteomalacie eine Rolle spielen können. Ich habe darüber anläBlich der Besprechung der mono- und pluriglandulären Störungen, bzw. Grundlagen dieser Osteopathien berichtet ${ }^{2}$ ). Es sei unter meinen Beobachtungen besonders auf einen Fall von Osteomalacie bei einer dauernd amenorrhoischen Fettsüchtigen mit infantilen Genitalien hingewiesen, die später an Osteomalacie, Myxödem wechselnd mit Basedowsymptomen und Tetanje, also ausgesprochener pluriglandulärer Insuffizienz, erkrankte, und auf einen weiteren von Rachitis tarda, die, eine geborene Hypoplastica von nur $140 \mathrm{~cm}$ Länge, ebenfalls konstitutionell hypo- oder amenorrhoisch und zeitlebens völlig frigide war.

Ich hoffe also gezeigt zu haben, wieviel größer die Bedeutung kongenitaler Hypo- und Dysplasien im Rahmen mannigfacher endokrin bedingter Krankheitsbilder ist, als die Mitteilung Krabbes anzunehmen schien.

1) Dtsch. Zeitschr. f. Nervenheilk., voraussichtlich Januar 1921.

2) Dtsch. Arch. f. klin. Med. 129, H. 1 u. 2. 